\title{
Longitudinal Motion Control of a High-Speed Supercavitation Vehicle
}

\author{
Bálint Vanek (vanek@aem.umn.edu)*, József Bokor \\ (bokor@aem.umn.edu) ${ }^{\dagger}$ and Gary J. Balas (balas@aem.umn.edu) \\ Aerospace Engineering and Mechanics, 110 Union St. SE, University of \\ Minnesota, Minneapolis, MN 55455
}

Roger E.A. Arndt (arndt001@umn.edu)

Civil Engineering and St. Anthony Falls Laboratory, 2 Third Ave. SE, University of Minnesota, Minneapolis, MN 55414

June 15, 2006

Abstract. This article focuses on theoretical developments in modeling and control of High-Speed Supercavitating Vehicles (HSSV). A simplified model of longitudinal dynamics is developed for control, and a dynamic inversion based inner-loop control technique is proposed to handle the switched, time-delay dependent behavior of the vehicle. Two outer-loop control schemes are compared for guidance level tracking. Various aspects of disturbance characteristics and actuator dynamics are investigated and analyzed.

Keywords: supercavitation, dynamic inversion, bimodal systems, receding horizon control

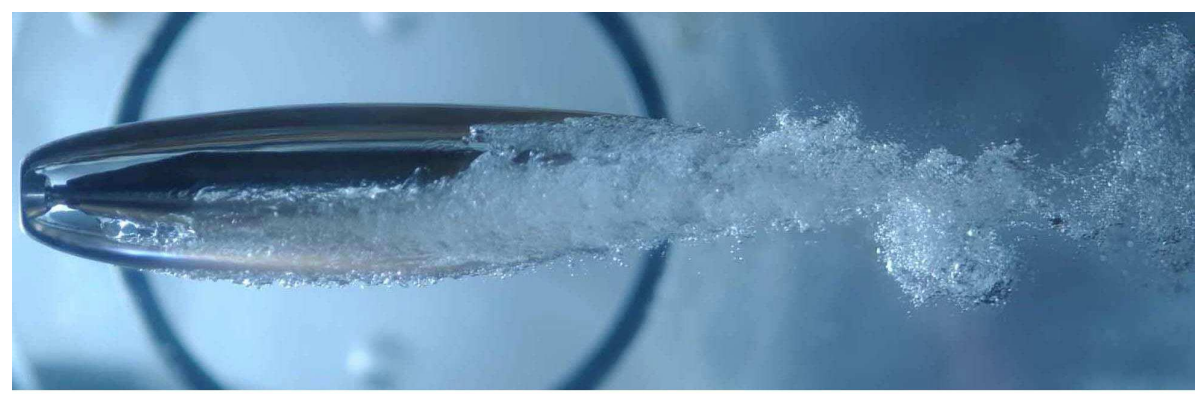

Figure 1. Water tunnel experiment on supercavitation

\section{Introduction}

Cavitation is an undesired phenomena in most engineering applications. Recent developments in supercavitation, motivated by the demand for

* corresponding author

$\dagger$ on sabbatical leave from MTA SZTAKI, Hungarian Academy of Sciences, Budapest XI. Kende u. 13-17, H-1518 Budapest, POB 63, Hungary

(C) 2006 Bálint Vanek. University of Minnesota. 
high-speed underwater vehicles (DARPA Advanced Technology Office, 2005; Ashley, 2001) has generated renewed interest in cavitation. Supercavitation can provide significant benefit for drag reduction by maintaining a stable single vaporized water bubble around the vehicle making it possible to extend the velocity range of underwater applications.

The sharp edge of a cavitator mounted on the nose of the vehicle creates large pressure gradient at high velocity. This phenomena leads to flow separation, which can create a cavity bubble encompassing the entire body. Only small regions of the vehicle are in contact with water. The cavitator and fins at the aft end (if present) provide lift to stabilize the body and may be used for guidance level control tasks. The cavitator size, shape and forward velocity determine the dimensions of the cavity bubble in which the body must fit. Any other contact with fluid phase is undesirable from a drag reduction standpoint. In this vehicle configuration, with no additional lift components, the vehicle is unstable inside the cavity. One means to address the vehicle stability involves the body itself. When the aft end penetrates the cavity surface, a large restoring force, known as planing, forces the vehicle back inside the cavity. Planing can be used as a support force to improve maneuverability and eliminate the need for fins. This leads to a reduced cost and system complexity, but it can also lead to limit cycle oscillations and increased drag if not controlled.

The actuation of the cavitator mounted on the nose becomes problematic if the goal is not only longitudinal control but three dimensional trajectory tracking. The influence of high cavitator actuation rate on the cavity stability and shape is uncertain. If planing does not support the vehicle weight, then fins at the aft end have to provide a lift force to balance the moment and stabilize the pitch motion. The fins effectiveness is a nonlinear function of their immersion depth, and planing can also occur with actively controlled fins. Note that the gap between the body and the cavity wall is on the order of $1-5 \mathrm{~cm}$ compared to the body length of $1-2$ meters, for vehicle speeds between 75 and $100 \mathrm{~m} / \mathrm{s}$. Cavity-vehicle interaction also has memory effects since the cavity shape, which affects planing, is a function of the vehicle past path and buoyancy. Suppression of limit cycle oscillations and disturbance attenuation require high bandwidth actuators, precise inertial measurements, and high sample rate real-time control algorithms.

The Russian navy has a supercavitation vehicle, the Shkval, currently in operation (Ashley, 2001), and German Diehl BGT Defence has demonstrated successful stable, straight and curved path maneuvers with the Barracuda (www.diehl-bgt-defence.de) supercavitating vehicle. Despite these successes, no solution to the three dimensional 
trajectory tracking problem for supercavitating vehicles is available in the open literature. Significant research on supercavitation vehicle control has been done in the past years, mostly using linear regulators ((Shao et al., 2003; Goel, 2002; Kirschner et al., 2002b; Kurdila et al., 2003)). These model-based designs rely on linearized dynamics around operating points. None of these approaches provide a planing-free operation which is a potential requirement in the future Underwater Express program ((DARPA Advanced Technology Office, 2005)). Other control approaches, using nonlinear techniques ((Dzielski and Kurdila, 2003; Lin et al., 2004)) are in the early development phase. Numerous challenges face the designers of supercavitating vehicle control systems.

This paper focuses on control challenges associated with the longitudinal axis motion of supercavitating vehicles. The first part of the paper (Section 2) describes a simplified pitch-plane model of a HSSV. This model is a refined version of the model described in (Vanek et al., 2006a), which was motivated by the papers of Dzielski and Kurdila (2003) and Kirschner et al. (2003). The model includes delay-dependent interaction between the vehicle and cavity wall, pitch-angle-dependent terms, and a refined planing model. In contrast with previous models, the equations of motion are written around the center of gravity. The second part of the paper summarizes the theoretical results on bimodal systems, which enable the systematic design of a dynamicinversion-based inner-loop control architecture (Section 3). An outerloop controller is added to guarantee high-level tracking objectives. In this paper, a pole-placement trajectory tracking controller with vertical position and pitch-angle-tracking objectives is compared with a receding horizon control (RHC) based design, where position and angle tracking, planing avoidance, and actuator saturation are formulated as performance objectives.

This paper adds to the literature by adressing planing avoidance as a performance objective. Linear control methods previously proposed ((Goel, 2002),(Shao et al., 2003)) did not take into account the large deviations from the nominal equations of motion, hence stability and performance were not guaranteed when the vehicle was planing. Nonlinear methods proposed by ((Dzielski and Kurdila, 2003),(Vanek et al., 2006b)) guarantee stability during planing, using the control surfaces to attenuate the large forces caused by planing, but do not take into account actuator position and rate limits, which restricts the operation envelope of the vehicle. The result of this research show that planing avoidance as a performance objective can significantly expand the operation envelope of the HSSV. The increased performance comes at the expense of slightly degraded position tracking performance, while planing with undesirably high acceleration happens seldom if ever. 
Simulation results of the closed-loop systems are compared in Section 5. Challenges facing the control designer are highlighted with respect to the actuator and sensor requirements, modeling issues, vehicle configuration, robustness and performance. Conclusions and future directions emphasizing the most important design criteria are highlighted in the last section.

\section{Mathematical Model}

Several mathematical descriptions are available in the literature of supercavitating vehicles, ranging from vertical directional one degreeof-freedom (DOF) model (Kirschner et al., 2003), through a simplified 2-DOF longitudinal description (Dzielski and Kurdila, 2003) to a high fidelity 6-DOF model (Kirschner et al., 2002a). A broader overview on the characteristics of these models can be found in (Balas et al., 2006) where another 2-DOF model from (Vanek et al., 2006a) is described.

Delay-dependent behavior of the cavity with refined treatment of planing was developed in (Vanek et al., 2006b), however it neglected the control forces' pitch angle dependence. Given a single control surface an additional force to support the vehicle requires constant nonzero pitch angle of the vehicle. Hence, a relationship between the pitch angle, moments and forces need to be developed. This is apparent since trimming the vehicle around straight level flight requires nonzero angle of attack on the fins and cavitator, to generate the required force and moment balance.

For simplicity, the equations of motion are written around the center of gravity (c.g.) and small angle approximations are used to eliminate trigonometric nonlinearities. The small angle assumption is valid since we anticipate angles less than $0.2 \mathrm{rad}$. Variable definition and coordinate directions are shown in Figure 2. The geometry of the model is intended to capture the main characteristics of a test vehicle. The body consists of a cylindrical and a conical section, with the length of the latter half of the former. The reference coordinate system is placed at the center of gravity with positive $x$-axis pointing in forward horizontal direction and the $z$-axis pointing to the center of Earth. The pitch angle is denoted by $\theta(\mathrm{rad})$, pitch rate $q(\mathrm{rad} / \mathrm{s})$, the vertical position $z(\mathrm{~m})$ and vertical velocity is $w(\mathrm{~m} / \mathrm{s}) . \delta_{c}(\mathrm{rad})$ is the cavitator angle with respect to the $x$ body axis, and $\delta_{f}(\mathrm{rad})$ the fin angle of attack in the body coordinates. In general, there are four forces acting on the body, the cavitator and fins forces, gravity, and planing which is not always present. 
The body length is denoted by $L(m)$ and its radius is $R(m)$. The body has uniform density $\rho_{b}=\rho m\left(\mathrm{~kg} / \mathrm{m}^{3}\right)$, with relative density $m$ compared with water $(\rho)$, from which the mass and inertia can be calculated, neglecting the cavitator and fins contribution. Hence, the vehicle mass $(M)$, moment of inertia around the y axis $\left(I_{y y}\right)$ and center of gravity location from the nose $\left(x_{c g}\right)$ are given as:

$$
\begin{gathered}
M=\frac{7}{9}(m \rho \pi) R^{2} L \\
I_{y y}=\frac{11}{60} R^{4} L \pi \rho m+\frac{1933}{45360} R^{2} L^{3} \pi \rho m \\
x_{c g}=\frac{17}{28} L
\end{gathered}
$$

If the full vehicle body is inside the cavity, hydrodynamic forces only

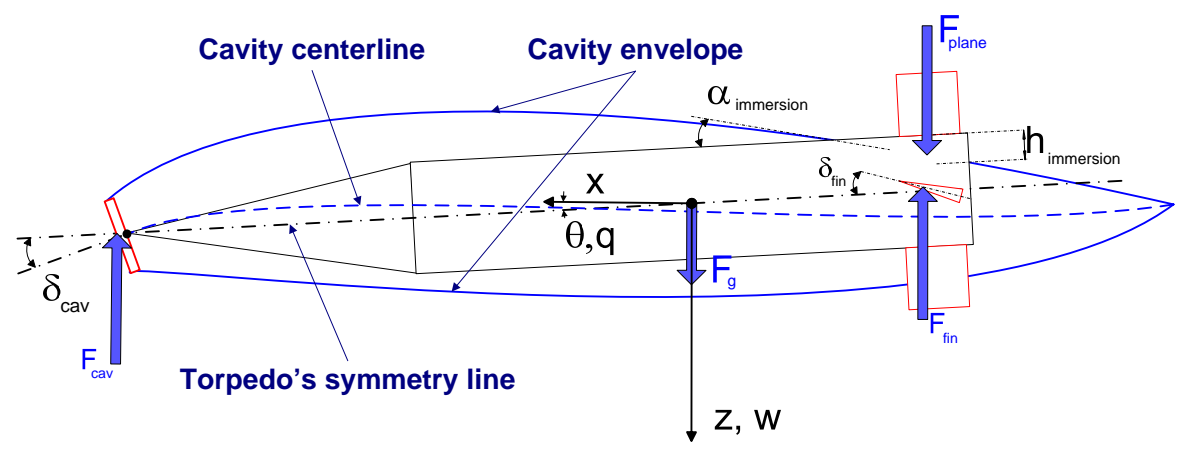

Figure 2. Variables in the longitudinal plane

act on the cavitator and the fins. The cavitator drag coefficient is modeled as $C_{x}=C_{x_{0}}(1+\sigma)$ where $\sigma$ is the cavitation number and $C_{x_{0}}=0.82$ (Logvinovich, 1972). The resulting lift on the cavitator is approximately:

$$
F_{\text {cav }}=\frac{1}{2} \pi \rho R_{n}^{2} V^{2} C_{x} \alpha_{c}=C_{l} \alpha_{c}
$$

where $R_{n}(m)$ is the cavitator radius, $\rho$ the water density, $V(\mathrm{~m} / \mathrm{s})$ the vehicle's horizontal speed, and $\alpha_{c}$ is the cavitator angle of attack. The force acting on the fins located at the tail is modeled as:

$$
F_{f i n}=n C_{l} \alpha_{f}
$$

where $n$ represents the fins effectiveness in providing lift as a function of angle of attack $\left(\alpha_{f}\right)$ relative to the cavitator. Note that in the longitudinal plane, the two horizontal fins are assumed to move in unison. Only 
small angle deflections with maximum value of $0.2 \mathrm{rad}$ are considered. The following simplifying assumptions are assumed through out this paper: the horizontal velocity $(V)$, water density $(\rho)$ and cavitation number $(\sigma)$ are assumed constant.

The force and moment equations around the c.g. using the conventions shown in Figure 2 are written as:

$$
\begin{gathered}
M \dot{w}=F_{c a v}+F_{f i n}+F_{g}+F_{p} \\
I_{y y} \dot{q}=-\left(F_{c a v} L_{c}+F_{f i n} L_{f}+F_{p} L_{f}\right)
\end{gathered}
$$

where $L_{c}=17 / 28 L$ and $L_{f}=-11 / 28 L$ are the respective moment arms of the cavitator and fin forces. The planing force is assumed to act at $-L_{f}$ distance from the c.g. The force components as function of vehicle states are:

$$
\begin{gathered}
F_{\text {cav }}=\frac{1}{2} \pi \rho R_{n}^{2} V^{2} C_{l}\left(\frac{w}{V}-\frac{q L_{c}}{V}+\theta+\delta_{c}\right) \\
F_{\text {fin }}=-\frac{1}{2} \pi \rho R_{n}^{2} V^{2} C_{l} n\left(\frac{w}{V}-\frac{q L_{f}}{V}+\theta+\delta_{f}\right) \\
F_{g}=\frac{7}{9} \rho m \pi R^{2} L g
\end{gathered}
$$

The remaining term $F_{p}$ associated with the planing force needs further consideration. This force is present when the vehicle transom interacts with the cavity wall, leading to a force similar to that sustained by powerboats bouncing on the top of the water. In the particular case of the HSSV, the free fluid surface is the circular cavity wall created by the cavitator and the vehicle tail is tubular. Logvinovich (1972) and Vasin and Paryshev (2001) analyzed the situation when a cylindrical body immerses into a cylindrical free fluid surface, which applies to the current vehicle configuration. These analytical results relate the immersion depth $h(m)$ and the distance from the axis of symmetry to the narrowest part of the spray sheet, generated by the displaced fluid.

The pressure force on the body is calculated from the energy of the spray sheet. If the diameter of the cavity at the planing location is $R_{c}$, then for $\left(R_{c}-R\right)<<R_{c}$ and small immersion angles, the planing force can be approximated as:

$$
F_{p}=-\rho R^{2} \pi V^{2}\left(1-\frac{R^{\prime}}{h^{\prime}-R^{\prime}}\right)^{2}\left(\frac{1+h^{\prime}}{1+2 h^{\prime}}\right) \alpha_{p}
$$

The variable $R^{\prime}$ denotes the normalized difference between the cavity and body diameter $\left(R^{\prime}=\left(R_{c}-R\right) / R\right)$. The variables $h^{\prime}$ the normalized immersion depth and $\alpha_{p}$ (rad) the immersion angle, capture 
the switched, nonlinear behavior of the dynamics. From Figure 2, the planing depth is determined by the cavity shape (as a function of the cavitator trajectory), vehicle position, and orientation. The position of the vehicle transom, where planing occurs, is a function of the vehicle position, rotation, and vehicle radius at the transom.

The cavity boundary is located at $R_{c}$ distance from its centerline, which is determined by the vehicle nose path through the water. At the transom region, the centerline is at $z_{n}(t-\tau)$. The cavity radius at the planing location is assumed to be constant. Hence, the immersion depth is the difference between the two quantities, and the nose position $z_{n}(t)=z(t)-L_{c} \theta(t)$.

$h^{\prime}=\left\{\begin{array}{lll}\frac{1}{R}\left[z(t)+\theta L_{f}+R-z_{n}(t-\tau)-R_{c}\right] & \text { if } & z_{n}(t-\tau)+R_{c}<z(t)+\theta L_{f}+R \\ 0 & \text { (inside cavity) } \\ \frac{1}{R}\left[z_{n}(t-\tau)-R_{c}-z(t)-\theta L_{f}+R\right] & \text { if } & z_{n}(t-\tau)-R_{c}>z(t)+\theta L_{f}+R\end{array}\right.$

Following the same reasoning, the immersion angle can be calculated based on the knowledge of the delayed vertical speed of the vehicle nose $\left(\dot{z}_{n}(t)=w(t)-V \theta(t)-L_{c} q(t)\right)$ and current pitch angle plus the contraction rate of the cavity bubble $\left(\dot{R}_{c}\right)$ :

$$
\alpha_{p}= \begin{cases}\theta-\frac{\dot{z}_{n}(t-\tau)+\dot{R}_{c}}{V} & \text { bottom contact } \\ 0 & \text { inside cavity } \\ \theta-\frac{\dot{z}_{n}(t-\tau)-\dot{R}_{c}}{V} & \text { top contact }\end{cases}
$$

It is important to note that, based on equation (12) the system is described by three different set of equations corresponding to three possible modes, one with linear and the other two with nonlinear delay dependent terms. The vehicle dynamics are continuous on the switching surface between different modes, since the nonlinear planing force is zero on the boundary.

Stability and hysteresis tests with a cavity-piercing fin performed in the high-speed water tunnel at St. Anthony Falls Laboratory (Syrstad et al., 2005) confirm the cavity shape specification as described in (Logvinovich, 1972). To simplify further the dynamic equations based on the assumption that the body planes at the approximate location of the fins, the following constants are defined:

$$
\kappa_{1}=\frac{L}{R_{n}}\left(\frac{1.92}{\sigma}-3\right)^{-1}-1, \quad \kappa_{2}=\left(1-\left(1-\frac{4.5 \sigma}{1+\sigma}\right) \kappa_{1}^{\frac{40}{17}}\right)^{\frac{1}{2}}
$$


Using the above expressions, the radius of the cavity at L distance from the cavitator is:

$$
R_{c}=R_{n}\left(0.82 \frac{1+\sigma}{\sigma}\right)^{\frac{1}{2}} \kappa_{2}
$$

The expression for the contraction rate of the cavity $\left(\dot{R}_{c}\right)$ is:

$$
\dot{R}_{c}=-\frac{20}{17}\left(0.82 \frac{1+\sigma}{\sigma}\right)^{\frac{1}{2}} V \frac{\left(1-\frac{4.5 \sigma}{1+\sigma} \kappa_{1}^{\frac{23}{17}}\right)}{\kappa_{2}\left(\frac{1.92}{\sigma}-3\right)}
$$

The equations for the cavity shape presented in equation (16) are valid given that they are evaluated sufficiently far from the cavitator:

$$
L>R_{n}\left(\frac{1.92}{\sigma}-3\right)
$$

but before the cavity closure.

Using these equations, supplemented by the basic kinematic equations for position and pitch angle:

$$
\dot{\theta}=q, \quad \dot{z}=w-V \theta
$$

the system states $z, \theta, w$ and $q$ can be written in state space form as:

$$
\left[\begin{array}{c}
\dot{z} \\
\dot{\theta} \\
\dot{w} \\
\dot{q}
\end{array}\right]=A\left[\begin{array}{c}
z \\
\theta \\
w \\
q
\end{array}\right]+B\left[\begin{array}{l}
\delta_{e} \\
\delta_{c}
\end{array}\right]+F_{\text {grav }}+F_{\text {plane }}(t, \tau)
$$

where $A$ and $B$ represent the linear part, $F_{\text {grav }}$ is a constant term and $F_{\text {plane }}$ corresponds to the nonlinear relationship associated with planing. The specific values of the system matrices are as follows:

$$
\begin{gathered}
A=\left[\begin{array}{cccc}
0 & -V & 1 & 0 \\
0 & 0 & 0 & 1 \\
0 & \frac{\left(C_{1}-C_{2}\right)}{M} & \frac{\left(C_{1}-C_{2}\right)}{(M V)} & \frac{\left(-C_{1} L_{c}+C 2 L_{f}\right)}{(M V)} \\
0 & \frac{\left(-C_{1} L_{c}+C_{2} L_{f}\right)}{I_{y y}} & \frac{\left(-C_{1} L_{c}+C_{2} L_{f}\right)}{\left(I_{y y} V\right)} & \frac{\left(C_{1} L_{c}^{2}-C_{2} L_{f}^{2}\right)}{\left(I_{y y} V\right)}
\end{array}\right] \\
B=\left[\begin{array}{cc}
0 & 0 \\
0 & 0 \\
\frac{C_{1}}{M} & \frac{C_{2}}{M} \\
\frac{-C_{1}}{I_{y y}} L_{c} & \frac{C_{2}}{I_{y y}} L_{f}
\end{array}\right] \\
F_{\text {grav }}=\left[\begin{array}{l}
0 \\
0 \\
g \\
0
\end{array}\right]
\end{gathered}
$$


Table I. System parameters for simulation model

\begin{tabular}{ccc}
\hline Parameter & Description & Value and Units \\
\hline$g$ & Gravitational acceleration & $9.81 \frac{\mathrm{m}}{\mathrm{s}^{2}}$ \\
$m$ & Density ratio, $\frac{\rho_{m}}{\rho}$ & 2 \\
$n$ & Fin effectiveness & 0.5 \\
$R_{n}$ & Cavitator radius & $0.0191 \mathrm{~m}$ \\
$R$ & Vehicle radius & $0.0508 \mathrm{~m}$ \\
$L$ & Length & $1.80 \mathrm{~m}$ \\
$V$ & Velocity & $75 \frac{\mathrm{m}}{\mathrm{s}}$ \\
$\sigma$ & Cavitation number & 0.03 \\
$C_{x 0}$ & Lift coefficient & 0.82 \\
\hline
\end{tabular}

$$
F_{\text {plane }}=\left[\begin{array}{c}
0 \\
0 \\
\frac{C_{p}}{M} \\
-\frac{C_{p}}{I_{y y}} L_{f}
\end{array}\right]\left(1-\frac{R^{\prime}}{h^{\prime}(t, \tau)-R^{\prime}}\right)^{2}\left(\frac{1+h^{\prime}(t, \tau)}{1+2 h^{\prime}(t, \tau)}\right) \alpha_{p}(t, \tau)
$$

The constant terms $C_{1}, C_{2}, C_{p}$ are defined to simplify the presentation. Their specific values are:

$$
C_{1}=\frac{1}{2} \pi \rho R_{n}^{2} V^{2} C_{x}, C_{2}=\frac{1}{2} \pi \rho R_{n}^{2} V^{2} C_{x} n, C_{p}=\pi \rho R^{2} V^{2}
$$

The system parameters are based on the benchmark HSSV in (Dzielski and Kurdila, 2003) and presented in Table I.

\section{Theoretical aspects of controller design}

The state space equations describing the system in equation (19) represent a bimodal switched system. Several characteristics of this model are of interest: (i) in the first mode, the system dynamics are linear (inside cavity), and in the second mode they are nonlinear (planing) input affine, though the control inputs affect the dynamics linearly in both modes, (ii) the switching condition does not depend on the control inputs, and (iii) the switching hyperplane depends on the delayed output variable $z_{n}(t-\tau)$.

A switched, hybrid control strategy was developed in Balas et al. (2006) for this type of system. Properties (i) and (ii) allow for feedback 
linearization in both modes. This is performed via a coordinate system with suitable geometric structure for the problem. It was shown (Balas et al., 2006) that this design results in linear dynamics in both modes ensuring continuous dynamics on the switching hypersurface. Since the latter depends on delayed state variables, controllability has to be analyzed, and a controller has to be designed that ensures stability and tracking performance.

The proposed approach relies on the assumption that the delay in the equations of motion can be eliminated by applying a suitable feedback. The resulting controllability analysis and control design can then be performed for bimodal linear time invariant (LTI) systems.

New state variables for equation (19) are selected for analysis and control design:

$$
\left[\begin{array}{l}
\bar{x}_{1}(t) \\
\bar{x}_{2}(t) \\
\bar{x}_{3}(t) \\
\bar{x}_{4}(t)
\end{array}\right]=\left[\begin{array}{c}
z_{n}(t) \\
-V \theta(t)+w_{n}(t) \\
\theta(t) \\
q(t)
\end{array}\right]
$$

The matrix used for this coordinate transformation is:

$$
T_{c}=\left[\begin{array}{c}
c_{1} \\
c_{1} A \\
c_{2} \\
c_{2} A
\end{array}\right]=\left[\begin{array}{cccc}
1 & -L_{c} & 0 & 0 \\
0 & -V & 1 & -L_{c} \\
0 & 1 & 0 & 0 \\
0 & 0 & 0 & 1
\end{array}\right]
$$

The state space equations in the new coordinate system are:

$$
\dot{\bar{x}}= \begin{cases}A_{c} \bar{x}(t)+B_{c} u(t)+\bar{F}_{g} & \text { if } \bar{c}(\delta) \bar{x}(t) \leq 0, \\ A_{c} \bar{x}(t)+\bar{F}_{p}(t, x, \delta)+B_{c} u(t)+\bar{F}_{g} & \text { if } \bar{c}(\delta) \bar{x}(t) \geq 0,\end{cases}
$$

where

$$
A_{c}=\left[\begin{array}{cccc}
0 & 1 & 0 & 0 \\
-\alpha_{110} & -\alpha_{111} & -\alpha_{120} & -\alpha_{121} \\
0 & 0 & 0 & 1 \\
-\alpha_{210} & -\alpha_{211} & -\alpha_{220} & -\alpha_{221},
\end{array}\right] B_{c}=\left[\begin{array}{c}
0 \\
c_{1} A B \\
0 \\
c_{2} A B
\end{array}\right]
$$

The difference between $F_{\text {grav }}$ and $\bar{F}_{\text {grav }}$ is that $\bar{F}_{\text {grav }}=T_{c} F_{\text {grav }}+K_{1}$ where $K_{1}$ is a constant associated with the shift in the origin of the coordinate system. Similarly $\bar{F}_{\text {plane }}=T_{c} F_{\text {plane }}$.

The inputs enter linearly in the state equations in both modes, and it is assumed that all states can be measured. This allows us to select two outputs defined as $y_{1}=\bar{x}_{1}$ and $y_{2}=\bar{x}_{3}$, such that the vector 
relative degree is well defined in both modes, and in addition, they are identical, i.e. by defining:

$$
C_{c}=\left[\begin{array}{llll}
1 & 0 & 0 & 0 \\
0 & 0 & 1 & 0
\end{array}\right]
$$

The relative degree for the modes are:

$$
\begin{array}{llll}
r_{1}^{2}=2, & r_{2}^{1}=2, & r_{1}^{2}+r_{2}^{1}=n=4 & \text { Mode 1 } \\
r_{1}^{2}=2, & r_{2}^{2}=2, & r_{1}^{2}+r_{2}^{2}=n=4 & \text { Mode 2 }
\end{array}
$$

The consequence of this property is that one can apply state feedback in both modes to eliminate the time delay in Mode 1 and the nonlinearity (exact feedback linearization) in Mode 2 (Balas et al., 2005b). This feedback is given by (Balas et al., 2006):

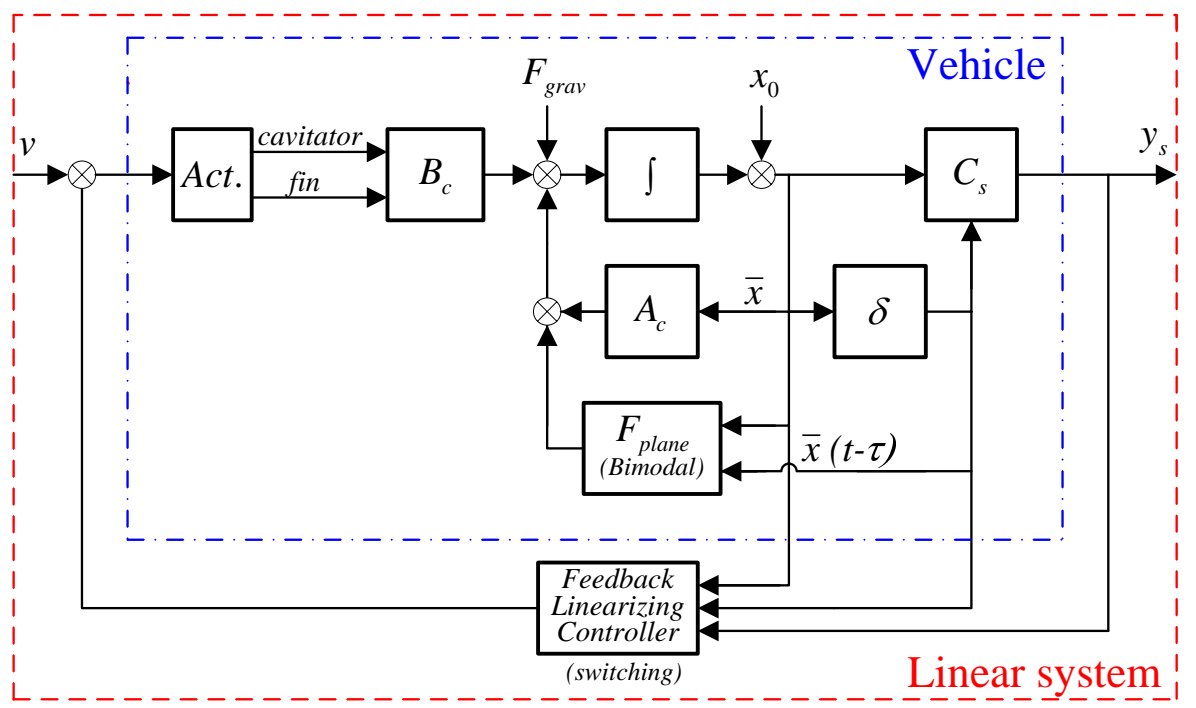

Figure 3. Control architecture for supercavitating vehicle model

$$
u_{f l c}= \begin{cases}M_{1}^{-1}\left(\dot{y}_{12, r e f}(t)-F_{\alpha} \bar{x}(t)-\bar{F}_{g}+v_{I}(t)\right) & \text { if } c(\delta) \bar{x}(t) \leq 0 \\ M_{1}^{-1}\left(\dot{y}_{12, r e f}(t)-F_{\alpha} \bar{x}(t)-\bar{F}_{g}-\bar{F}_{p}(x, \delta)+v_{I I}(t)\right) & \text { if } c(\delta) \bar{x}(t) \geq 0\end{cases}
$$

where $M_{1}=(C A B), y_{12, \text { ref }}=\left[y_{1}, y_{2}\right]_{r e f}^{T}$. The feedback gain $F_{\alpha}$ is defined by the controllability invariants $\alpha_{i j k}$ of the linear part of the system (equation (28)). The structure of the designed feedback system is shown in Figure 3. 
The feedback linearized closed-loop has the following form in both modes:

$$
\dot{\bar{x}}=\left[\begin{array}{llll}
0 & 1 & 0 & 0 \\
0 & 0 & 0 & 0 \\
0 & 0 & 0 & 1 \\
0 & 0 & 0 & 0
\end{array}\right] \bar{x}+\left[\begin{array}{ll}
0 & 0 \\
1 & 0 \\
0 & 0 \\
0 & 1
\end{array}\right]\left[\begin{array}{l}
v_{1} \\
v_{2}
\end{array}\right]
$$

The switching condition is given by the sign of $y_{s}=c(\delta) \bar{x}$.

\subsection{Controllability analysis of the Bimodal System}

Controllability of linear bimodal systems with single input and dynamics continuous on the switching surface is analyzed in (Çamlibel et al., 2004). These results are generalized to multi-input systems in (Balas et al., 2005b) and applied to the supercavitating vehicle in (Balas et al., 2006). The results show that the controllability analysis can be performed by analyzing the controllability of the zero dynamics of the switched system with respect to the switching hyperplane $(\mathcal{C})$.

It can be shown that every linear system with relative degree $(r)$ and switching output $\xi_{1}=y_{s}$ has the following decomposition by applying state transform and state feedback:

$$
\begin{aligned}
\dot{\xi} & =A_{11} \xi+\gamma v \\
u_{p} & =\frac{1}{\gamma}\left(-A_{12} \eta-\bar{B}_{21} \bar{u}+v\right) \\
\dot{\eta} & =A_{22} \eta+\bar{B}_{22} \bar{u}+G y_{s},
\end{aligned}
$$

Since $\xi_{1}=y_{s}$, equation (36) describes the dynamics of the system on $\mathcal{C}$. Rewrite the equation of this zero dynamics as:

$$
\dot{\eta}=P \eta+Q \bar{u}+R y_{s} .
$$

It is proved in (Balas et al., 2005b) that either the pair $(P, Q)$ has to be controllable, then $\eta$ is controllable "without" using $y_{s}$, e.g. by applying $\bar{u}=Q^{\#}\left(-R y_{s}+w\right)$, or if the pair $(P, Q)$ is not controllable, then the conditions of controllability of the bimodal switching system reduces to the controllability of equation (37) using unconstrained $\bar{u}$ and nonnegative (or in the other mode non-positive) input $y_{s}$ : (i) The pair $(P,[Q R])$ has to be controllable, (ii) Consider the decomposition of equation (37) induced by the reachability subspace $\mathcal{R}(P, Q)$,

$$
\begin{array}{lcc}
\dot{\eta}_{1}= & P_{11} \eta_{1}+P_{12} \eta_{2}+Q \bar{u}+R_{1} y_{s} \\
\dot{\eta}_{2}= & P_{22} \eta_{2}+R_{2} y_{s},
\end{array}
$$

where $R_{2} \neq 0$. Then the imaginary part of the eigenvalues of $P_{22}$ cannot be zero. 
For the high-speed supercavitating vehicle model, this result has to be generalized for a time delay system. Since only one delay time is present in the switching condition, it is possible to discretize the system using backward difference approximation (Safonov, 1987) that preserves the relative degree needed to analyze the zero dynamics, with extended state space by including the delayed state variable.

This results in the following discrete time state equations:

$$
x(t+1)=A_{d} x(t)+B_{d} v(t), \quad y_{s}=C_{d} x(t)
$$

where

$$
A_{d}=\left[\begin{array}{lllll}
1 & T & 0 & 0 & 0 \\
0 & 1 & 0 & 0 & 0 \\
0 & 0 & 1 & T & 0 \\
0 & 0 & 0 & 1 & 0 \\
1 & 0 & 0 & 0 & 0
\end{array}\right], \quad B_{d}=\left[\begin{array}{cc}
0 & 0 \\
\beta_{21} T & \beta_{22} T \\
0 & 0 \\
\beta_{41} T & \beta_{42} T \\
0 & 0
\end{array}\right], \quad C_{d}=[1,0, V, 0,-1]
$$

$T$ denotes the sample time and the fifth state is the delayed position of the cavitator. The switched output $\left(C_{d}\right)$ is derived based on equation (12). The above system can be decomposed into the following set of equations:

$$
\begin{gathered}
\xi(t+1)=\left[\begin{array}{cc}
0 & a_{12} \\
0 & a_{22}
\end{array}\right] \xi(t)+\left[\begin{array}{ccc}
0 & 0 & 0 \\
0 & b_{22} & b_{23}
\end{array}\right] \eta(t)+\left[\begin{array}{c}
0 \\
e_{21}
\end{array}\right] v_{1}(t)+\left[\begin{array}{c}
0 \\
e_{22}
\end{array}\right] v_{2}(t) \\
y_{s}=\left[\begin{array}{ll}
1 & 0
\end{array}\right] \xi(t) \quad \text { switching condition } \\
\eta(t+1)=P \eta(t)+R \xi(t)+Q v_{2}(t)
\end{gathered}
$$

where

$$
P=\left[\begin{array}{ccc}
p_{11} & p_{12} & p_{13} \\
0 & p_{22} & p_{23} \\
0 & 0 & p_{33}
\end{array}\right], \quad R=\left[\begin{array}{cc}
0 & r_{12} \\
0 & r_{22} \\
0 & 0
\end{array}\right], \quad Q=\left[\begin{array}{c}
0 \\
0 \\
q_{31}
\end{array}\right]
$$

The zero dynamics with respect to the first input $v_{1}$ are described by equation (44) and a similar decomposition can be obtained with respect to $v_{2}$. Using the results in (Balas et al., 2006), it can be seen that the $(P, Q)$, pair is controllable in both cases, implying that the dynamic inversion controller using inputs $v_{1}$ and $v_{2}$ with switching can be applied to control the bimodal system and an additional linear controller can be used for trajectory tracking. 


\section{Outer loop control strategy}

A single, linear outer-loop controller can guarantee stability and achieve the desired tracking properties with feedback linearization, since the system behaves the same regardless of the interior switching state. A variety of linear design approaches can be used for stability and control (Doyle et al., 1989; McFarlane and Glover, 1992; Balas et al., 2005a; Mayne et al., 2000). The ability of the controller to directly handle constraints could provide significant benefits if planing is restricted. Hence, a simple pole placement controller is compared with receding-horizon control approach which allows for actuator and state constraints.

The inner loop dynamics after feedback linearization, using the new canonical coordinates are:

$$
\left[\begin{array}{c}
\dot{x}_{1} \\
\dot{x}_{2} \\
\dot{x}_{3} \\
\dot{x}_{4}
\end{array}\right]=\left[\begin{array}{llll}
0 & 1 & 0 & 0 \\
0 & 0 & 0 & 0 \\
0 & 0 & 0 & 1 \\
0 & 0 & 0 & 0
\end{array}\right]\left[\begin{array}{l}
x_{1} \\
x_{2} \\
x_{3} \\
x_{4}
\end{array}\right]+B_{c}\left[\begin{array}{l}
\Delta \delta_{f} \\
\Delta \delta_{c}
\end{array}\right]
$$

where $\Delta \delta_{f, c}$ denotes the additional deflection of the fins and cavitator commanded by the higher level controller. This system is nilpotent, because all eigenvalues of $A$ are zero. There is no cross coupling between the first two states (vertical position and speed), and the other states (vehicle angle and angle rate). Hence, they can be controlled independently by the two control inputs.

\subsection{Multivariable Pole Placement for Tracking}

An easy and tractable control design approach for linear systems is pole placement. The performance objective is to track desired state commands with no restrictions on the maximum actuator deflections, since the tracking signals have minor contribution compared with the action due to planing. With the assumption of full state feedback, this can be done fairly simply. The inversion based controller has the form:

$$
\begin{aligned}
{\left[\begin{array}{l}
u_{1}(t) \\
u_{2}(t)
\end{array}\right]=(C A B)^{-1} } & \left(-\left[\alpha_{u}\right]\left[\begin{array}{l}
x_{1}(t) \\
x_{2}(t)
\end{array}\right]-\right. \\
& \left.-\left[\alpha_{l}\right]\left[\begin{array}{l}
x_{3}(t) \\
x_{4}(t)
\end{array}\right]-\left[G_{c}\right]-\left[P_{c}(t, \tau)\right]\right)+\left[\begin{array}{l}
\Delta \delta_{f} \\
\Delta \delta_{c}
\end{array}\right]
\end{aligned}
$$

Where the $\alpha_{u, l}$ coefficients are the elements of the $A_{c}$ matrix (equation (28)) and $\Delta \delta_{f, c}$ are the signals responsible for reference tracking.

$$
\left[\alpha_{u}\right]=\left[\begin{array}{ll}
-\alpha_{110} & -\alpha_{111} \\
-\alpha_{210} & -\alpha_{211}
\end{array}\right]\left[\alpha_{l}\right]=\left[\begin{array}{ll}
-\alpha_{120} & -\alpha_{121} \\
-\alpha_{220} & -\alpha_{221}
\end{array}\right]
$$


The reference tracking part of the controller responsible for pole locations:

$$
\begin{aligned}
{\left[\begin{array}{l}
\Delta \delta_{f} \\
\Delta \delta_{c}
\end{array}\right]=(C A B)^{-1} } & \left\{\left[\begin{array}{cc}
-\bar{\alpha}_{110} & -\bar{\alpha}_{111} \\
0 & 0
\end{array}\right]\left[\begin{array}{l}
x_{1}(t)-x_{1, r e f}(t) \\
x_{2}(t)-x_{2, r e f}(t)
\end{array}\right]+\right. \\
+ & {\left.\left[\begin{array}{cc}
0 & 0 \\
-\bar{\alpha}_{220} & -\bar{\alpha}_{221}
\end{array}\right]\left[\begin{array}{l}
x_{3}(t)-x_{3, r e f}(t) \\
x_{4}(t)-x_{4, r e f}(t)
\end{array}\right]+\left[\begin{array}{l}
\dot{x}_{1, r e f} \\
\dot{x}_{3, r e f}
\end{array}\right]\right\} }
\end{aligned}
$$

The feedback linearized closed loop has the following form in all modes:

$$
A_{c l}=A_{c}-B_{c} F_{i n v}+B_{c} F_{c t r}=\left[\begin{array}{cccc}
0 & 1 & 0 & 0 \\
-\bar{\alpha}_{110} & -\bar{\alpha}_{111} & 0 & 0 \\
0 & 0 & 0 & 1 \\
0 & 0 & -\bar{\alpha}_{220} & -\bar{\alpha}_{221}
\end{array}\right]
$$

The closed-loop system is stable for a given set of $\bar{\alpha}$ coefficients.

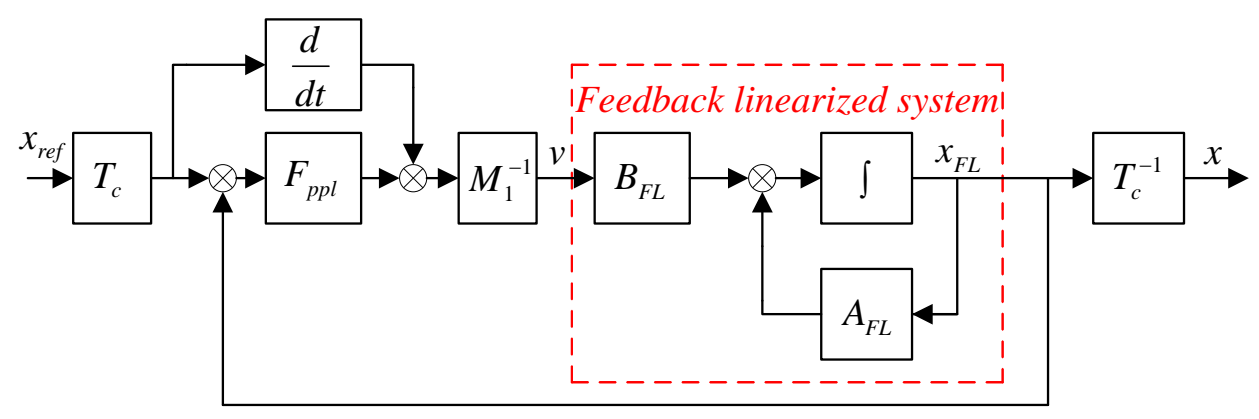

Figure 4. Control architecture for supercavitating vehicle model

The tracking part of the controller is responsible for the location of the poles. The eigenvalues of the system are:

$$
\begin{aligned}
& \lambda_{1,2}=-0.5 \bar{\alpha}_{221} \pm 0.5 \sqrt{\left(-\bar{\alpha}_{221}\right)^{2}-4 \bar{\alpha}_{220}} \\
& \lambda_{3,4}=-0.5 \bar{\alpha}_{121} \pm 0.5 \sqrt{\left(-\bar{\alpha}_{121}\right)^{2}-4 \bar{\alpha}_{120}}
\end{aligned}
$$

The poles can be freely adjusted in the stable region, while the driving factor for actuator deflections remains planing cancelation. Hence, the only limiting factor for setting the pole locations is the actuator bandwidth.

The structure of the feedback controller is shown in Figure 3. The inner-loop controller feedback linearizes the system, and the outer-loop 
controller handles reference tracking. It is possible to track both position and angle commands with consistent position, velocity, angle and angle rate reference signals. The designed pole placement controller also operates on the transformed canonic coordinates. The special structure of the feedback linearized system allows the vehicle position and angle to be controlled independently.

\subsection{Outer-Loop RHC CONTROL}

This section describes the design of an outer-loop controller using Receding Horizon Control (RHC). The previous section focused on the inner-loop control with a simple pole-placement controller to achieve reference tracking properties. In addition to the performance specifications, the reference tracking control should avoid actuator saturation and immersion into the fluid, preventing the inner loop to command unrealistically high deflections to cancel out the forces generated by planing. Predicting planing may provide beneficial information which can broaden the stable operation envelope of the vehicle, enabling more aggressive reference trajectories, at the expense of slightly degraded tracking performance.

A popular way to avoid saturations on the actuators is to use prediction based control methods (Receding Horizon Control or Model Predictive Control). The proposed control scheme is shown in Figure 5.

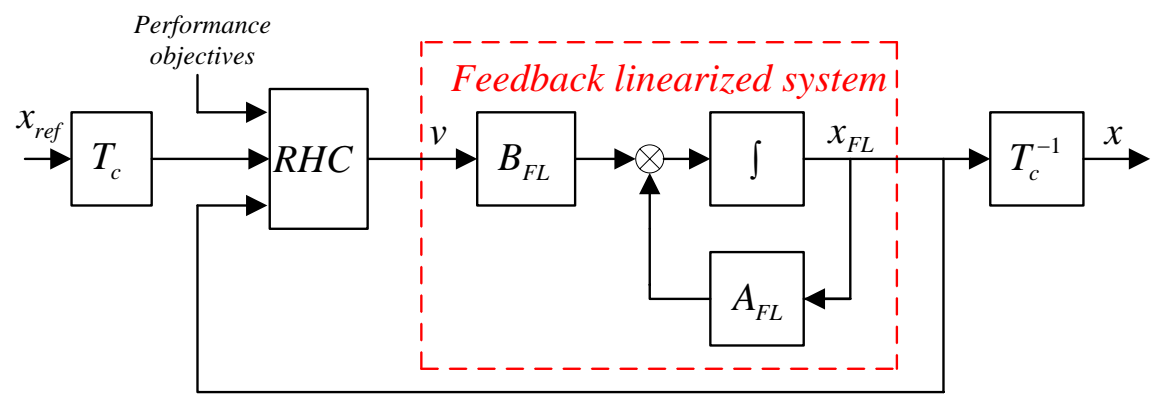

Figure 5. The RHC control loop structure

The controller structure differs from the controller discussed in (Balas et al., 2006), as the outer-loop uses RHC technique (equation 52).

$\left[\begin{array}{l}u_{1}(t) \\ u_{2}(t)\end{array}\right]=(C A B)^{-1}\left(-\left[\alpha_{u}\right]\left[\begin{array}{l}x_{1}(t) \\ x_{2}(t)\end{array}\right]-\left[\alpha_{l}\right]\left[\begin{array}{l}x_{3}(t) \\ x_{4}(t)\end{array}\right]-\left[G_{c}\right]-\left[P_{c}(t, \tau)\right]-\left[\begin{array}{l}v_{1, R H C}(t) \\ v_{2, R H C}(t)\end{array}\right]\right)$

The standard linear RHC problem and solution using quadratic programming is well known (Maciejowski, 2002) and reliable software tools 
are available for controller design (Bemporad et al., 2005). The discrete time controller is obtained in a receding horizon fashion using modelbased predictions by solving a finite time constrained optimization problem:

$$
\begin{array}{r}
\min _{\Delta u(k \mid k), \ldots, \Delta u(m+k-1 \mid k), \varepsilon}\left\{\sum _ { i = 0 } ^ { p - 1 } \left(\sum_{j=1}^{n_{y}}\left|w_{i+1, j}^{y}\left(y_{j}(k+i+1 \mid k)-r_{j}(k+i+1)\right)\right|^{2}+\right.\right. \\
\left.\left.+\sum_{j=1}^{n_{u}}\left|w_{i, j}^{\Delta u} \Delta u_{j}(k+i \mid k)\right|^{2}+\sum_{j=1}^{n_{u}}\left|w_{i, j}^{u}\left(u_{j}(k+i \mid k)-u_{j, \text { des }}(k+i)\right)\right|^{2}\right)+\rho_{\varepsilon} \varepsilon^{2}\right\}
\end{array}
$$

where $\Delta u$ denotes the input increments, $(k+i \mid k)$ indicates the value for time $k+i$ using the available information at $k$. The tracking is achieved by minimizing the error between $y(k+i \mid k)$ the predicted output and the reference $(r(k+i))$. The actuator usage and input rates are also weighted in the cost function with $w_{i, j}$ coefficients. The constraints on inputs, input rates, or outputs can be implemented as soft constraints:

$$
u_{j, \min }(i)-\varepsilon V_{j, \min }^{u}(i) \leq u_{j}(k+i \mid k) \leq u_{j, \max }(i)+\varepsilon V_{j, \max }^{u}(i)
$$

where $\varepsilon$ is the slack variable relaxed with weight $V_{j}^{u}$, which is heavily penalized in the cost function with $\rho_{\varepsilon}$. Normally input constraints are implemented as hard constraints while output constraints are softened to ensure feasibility when large disturbances are expected. The prediction $\left(n_{y}\right)$ and control horizons $\left(n_{u}\right)$ have large impacts on the solution and computational requirements. In general the prediction does not exactly match the system response. Hence, the best solution is often obtained by a suitable finite prediction horizon, while the decision variable (the control signal), is changed over a shorter horizon, and then held constant through the end of the prediction horizon.

The special structure of the inner-loop controller requires only a single linear RHC controller for the feedback linearized system described by equation (33). The objectives are reference tracking and planing avoidance. One of the main assumptions is constant horizontal speed, hence the delay is assumed constant. The delay in the simulation is $1.8 \mathrm{~m} /(75 \mathrm{~m} / \mathrm{s})=0.024 \mathrm{~s}$ which is included in the discrete time system model used for predictions in the RHC controller. The extended statespace system includes the delayed position of the nose in addition to the states described in equation (55). The sampling time of the RHC controller is set to $0.008 s$, three unit delays are required to express the 
desired state.

$$
\left[\begin{array}{l}
x_{1}(t) \\
x_{2}(t) \\
x_{3}(t) \\
x_{4}(t) \\
x_{5}(t) \\
x_{6}(t) \\
x_{7}(t)
\end{array}\right]=\left[\begin{array}{c}
z(t) \\
-V \theta(t)+w(t) \\
\theta(t) \\
q(t) \\
z(t-\tau) \\
z(t-2 \tau) \\
z(t-3 \tau)
\end{array}\right]
$$

The system matrices used for prediction are derived from the continuous time model using backward difference approximation (Safonov, 1987). This preserves the simple geometry of the equations, including the relative degree, and the dynamics are easier tractable, than with the simple zero-order hold equivalence transformation.

$$
A_{d}=\left[\begin{array}{lllllll}
1 & T & 0 & 0 & 0 & 0 & 0 \\
0 & 1 & 0 & 0 & 0 & 0 & 0 \\
0 & 0 & 1 & T & 0 & 0 & 0 \\
0 & 0 & 0 & 1 & 0 & 0 & 0 \\
1 & 0 & 0 & 0 & 0 & 0 & 0 \\
0 & 0 & 0 & 0 & 1 & 0 & 0 \\
0 & 0 & 0 & 0 & 0 & 1 & 0
\end{array}\right], \quad B_{d}=\left[\begin{array}{cc}
0 & 0 \\
\beta_{21} T & \beta_{22} T \\
0 & 0 \\
\beta_{41} T & \beta_{42} T \\
0 & 0 \\
0 & 0 \\
0 & 0
\end{array}\right]
$$

$T$ denotes the sample time in $A_{d}$ and $B_{d}$. The seventh state $\left(x_{7}\right)$ represents the delayed position of the nose. The planing condition is expressed using $x_{7}$ with the relation described in equation (12):

$$
R-R_{c} \leq C_{p} x_{d} \leq R_{c}-R, C_{p}=[1,0, L, 0,0,0,-1]
$$

This additional output can be used in the control predictions to constrain planing, causing the inner-loop to generate smaller control deflections.

Direct constraint fulfilment cannot be guaranteed because the two controllers act parallel (Figure 3) and only the RHC signals are constrained. This is only sufficient for a limited maneuver range. Usually as soon as the RHC command reaches its maximum value, the body hits the cavity wall or the tracking performance becomes poor. The wall impact results in oscillations and increased control deflections, while the drag on the hull also increases. 


\section{Control of a Supercavitating Vehicle Model}

Simulations are performed in the MATLAB/SIMULINK environment, and parameter dependencies are analyzed for comparison with a basic setup. The reference trajectory is an obstacle avoidance maneuver: the horizontal speed is constant $75 \mathrm{~m} / \mathrm{s}$ while the vehicle moves down $2 \mathrm{~m}$ and returns to continue its straight path within 1.2 seconds, as seen in Figure 6 . The initial trajectory is composed by four arcs approximated by B-splines to provide continuous, easily numerically differentiable functions. The additional reference signals are derived using further assumptions. The vertical position change $(\dot{z}(t))$ is caused by the vertical speed of the vehicle $(w(t))$ plus the longitudinal speed component's projection to the vertical plane $(-V \theta(t))$. Earlier results (Dzielski and Kurdila, 2003) suggested that $w$ is closely related to planing, hence it is desired to be kept small.

$$
w_{\text {ref }}(t)=0, \quad \theta_{\text {ref }}(t)=\frac{-1}{V} \dot{z}_{\text {ref }}(t)
$$

The pitch rate reference $\left(q_{r e f}(t)\right)$ can be calculated as $\dot{\theta}_{r e f}(t)$. It is assumed that the environment (static pressure and water density) remains constant during the maneuver. A $200 \mathrm{rad} / \mathrm{s}$ first order actuator model is included in the simulation, which was not considered in the control design. In addition to the model mismatch, the system is also affected by random disturbance, which is based on measurements derived from water tunnel experiments done in UMN St. Anthony Falls Laboratory (Arndt et al., 2005). The cavity wall disturbance is modeled as white noise passed through a $150 \mathrm{~Hz}$ second order low-pass filter (equation (59)). The cavity disturbance has a maximum $10 \%$ magnitude of nominal cavity gap. This disturbance by nature does not show up in all situations, if the transom is far enough from the cavity walls, it has no effect on the vehicle. But if the transom is close to the cavity surface and immersion occurs, the immersion depth will be determined not only by the vehicle states but also by the noisy cavity radius which has a randomly varying component. A non-smooth cavity represents a challenge as the cavity wall is the switching surface of the controller.

$$
G_{n}=\frac{0.1}{55.93}(R c-R) \frac{10^{6}}{s^{2}+2000+10^{6}}
$$

\subsection{Pole Placement simulation Results}

The performance specifications are to track trajectory reference commands while minimizing limit cycle oscillations. The reference tracking 

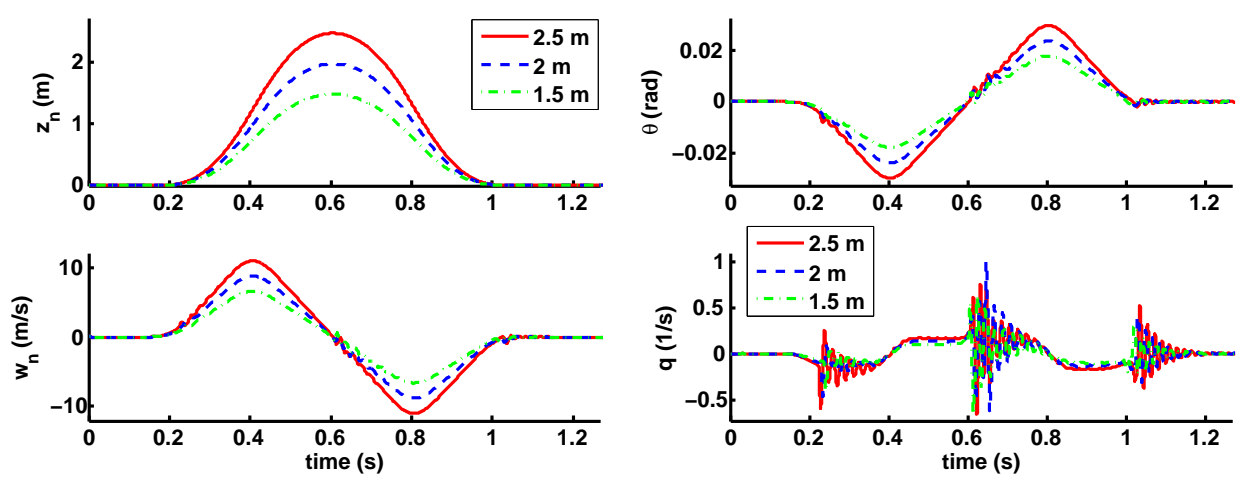

a. Translation

b. Rotation

Figure 6. Tracking different amplitude maneuvers with pole placement controller
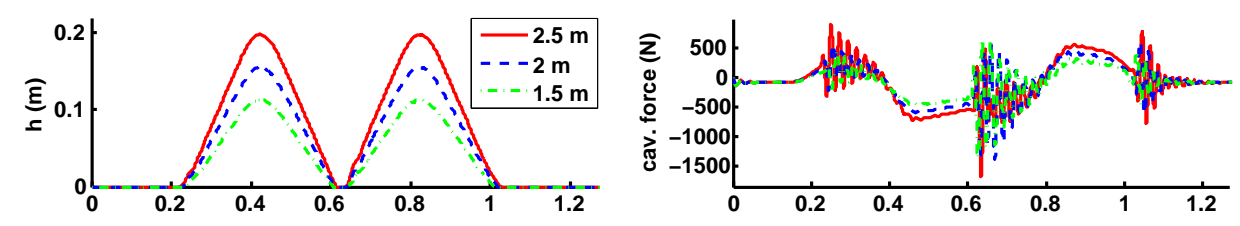

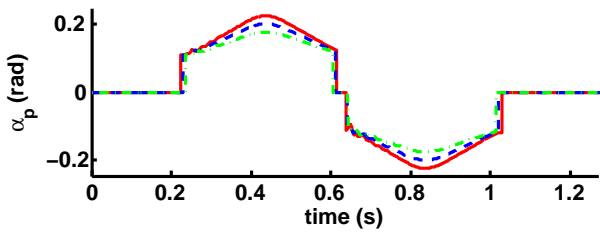

a. Planing

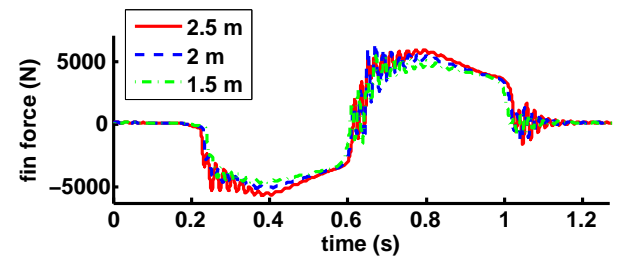

b. Actuator deflections

Figure \%. Tracking different amplitude maneuvers with pole placement controller

has lower priority compared with oscillation attenuation. The following controller gains were selected: $-\bar{\alpha}_{110}=-40000 ;-\bar{\alpha}_{111}=-400 ;-\bar{\alpha}_{220}=$ $-90000 ;-\bar{\alpha}_{221}=-600$. With which the resulting eigenvalues are $-300 ;-300 ;-200 ;-200$ (equation (51)).

The contribution from the tracking part of the controller with these high gains is still negligible compared with the inversion based contribution to compensate the effect of planing.

Three maneuvers with $1.5,2$ and $2.5 \mathrm{~m}$ amplitudes are compared in Figures 6 and 7. The tracking performance of the closed-loop system is very good, considering the high disturbance level, though the planing depth $(h)$ is significant. Large oscillations are present when the transom steps over from one mode to another. The planing depth has a clear relationship with the sharpness of the maneuver (Figure 7). These sharp maneuvers require large actuator deflections, and create significant drag. Increased thrust would be required to maintain con- 

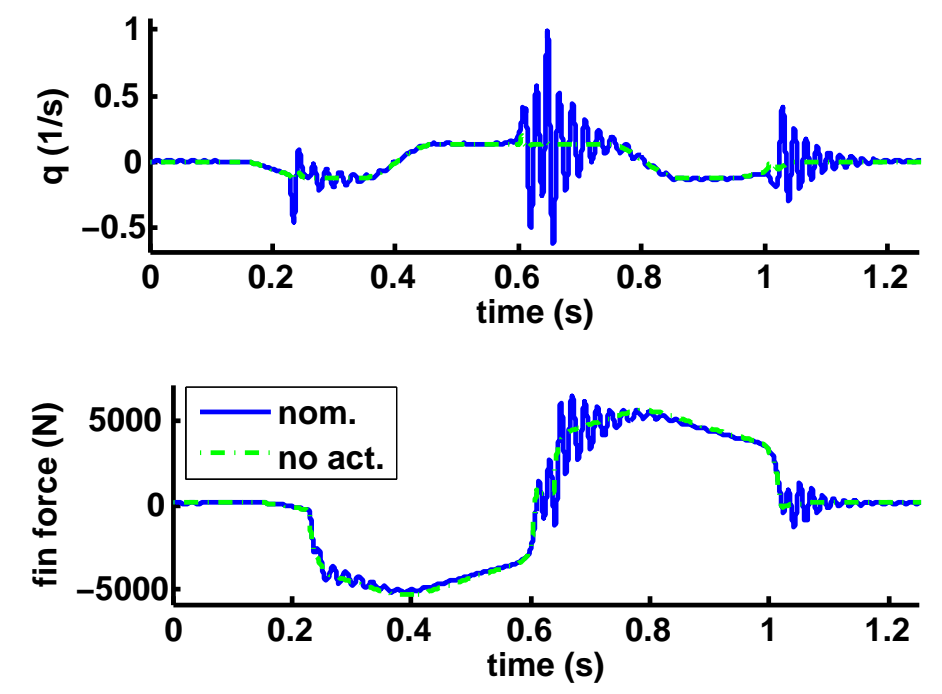

Figure 8. The actuator models role with pole placement controller

stant longitudinal speed. Including this additional control objective is undesirable, since thrust is not a control variable.

Accurate knowledge of delay in the cavity shape description plays an important role in the performance. The vehicle tracks the reference signal well given accurate information of the delay. Imprecise knowledge of the delay results in oscillations and the system becomes unstable at approximate $15-20 \%$ error. Simulations with $2.4 \mathrm{~ms}$ variation in the delay lead to poor performance with oscillations and intensive actuator usage.

The original controller was designed without an actuator model. The effect of a first order actuator with $30 \mathrm{~Hz}$ bandwidth is considered through the simulations. Significantly slower actuators were not able to stabilize the system, while faster actuators achieved better performance. The case when the actuator is treated as unity (Figure 8) clearly results in better performance than the one with the first order actuator model, since only small oscillations occur. All other results presented have the actuator model included.

Sensitivity to cavity wall disturbances is investigated by varying the magnitude and frequency content of the disturbance. The maximum planing depth remains the same if the disturbance magnitude increase by a factor of 5 to 0.5 times the cavity gap, but the actuator deflections are slightly more aggressive. The response has larger spikes and has longer settling times. Changing the second order disturbance filter to a first or third order filter with the same bandwidth has a small effect on the response. Hence the pole placement design is relatively insensitive 
to the smoothness of the cavity wall disturbance, because of the high planing depth.

The vehicle has noticeably different dynamical behavior for long excursion maneuver, which do not require high pitch rate motion, (Figure 12). The reference maneuver is a $4 s$ down-up maneuver with amplitude $20 \mathrm{~m}$, and as suggested in (Dzielski and Kurdila, 2003) with the reference on normal velocity $\left(w_{r e f}(t)\right)$ set to zero. The maneuver can be executed without planing, because the disturbances on cavity shape fade away noticeably faster than the maneuver changes. The cavity bubble is in quasi-steady state during the maneuver.

\subsection{RHC SimUlation RESUlts}

The continuous-time feedback linearization controller is implemented in the inner-loop while the discrete RHC controller is running at $0.008 s$ sampling time as an outer-loop (Figure 5). The predictive controller has a six step prediction horizon, which is sufficiently longer than the delay in the cavity description. The best results were achieved with a three step long control horizon, which allows sufficient freedom for the control solutions but is less sensitive to uncertainties in the predictions. Constraints are chosen corresponding to the physical limitations of the vehicle. The maximum actuator deflections are set to $\pm 0.2 \mathrm{rad}$ and the maximum deflection rates are $\pm 100 \mathrm{rad} / \mathrm{s}$. The maximum deflection is meant to constrain the maximum achievable force, while its angle value is less important, since the size of the fins are currently under investigation. The maximum vertical speed is $28.75 \mathrm{~m} / \mathrm{s}$ and the maximum pitch angle is set to $0.25 \mathrm{rad}$ to ensure the validity of small angle approximations. Structural loads are closely related to maximum pitch rate which is constrained to $\pm 10 \mathrm{rad} / \mathrm{s}$. Drag reduction and smooth motion with extending the operation envelope of the vehicle can be achieved with planing-free flight, while the control surface deflections are also lower. The maximum transom deviation from the cavity centerline is constrained to $1 \mathrm{~cm}$, which is smaller than the nominal cavity gap $(1.39 \mathrm{~cm})$ to guarantee planing avoidance in the presence of disturbances.

The optimization problem weights differently the input and output variables. The input weight is set to 100 on both inputs, and the input rate weight set to 50 . These weights can be interpreted with the knowledge of the output-error weights. The high position error weight (25000) indicates that position tracking received the highest priority, while the lower velocity error weight (1000), angle error weight (100) and angle rate error weight (2500) ensure that tracking of these variables have lower impact on the optimization. These slightly penal- 

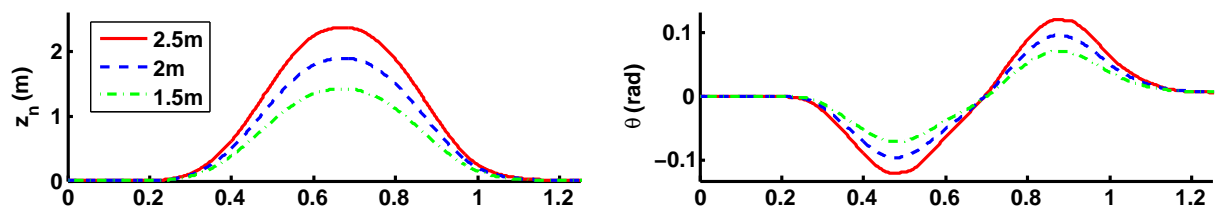

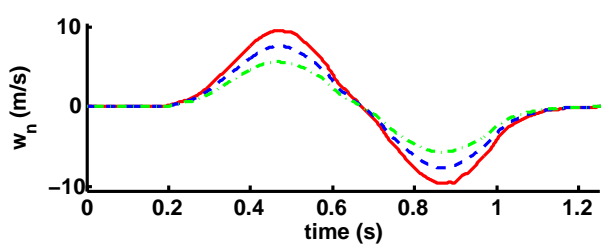

a. Translation

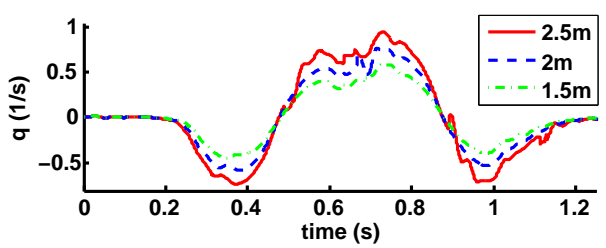

b. Rotation

Figure 9. Tracking different amplitude maneuvers with predictive controller
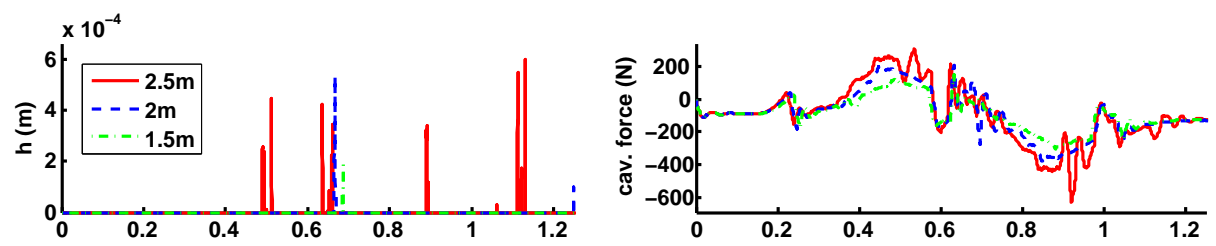

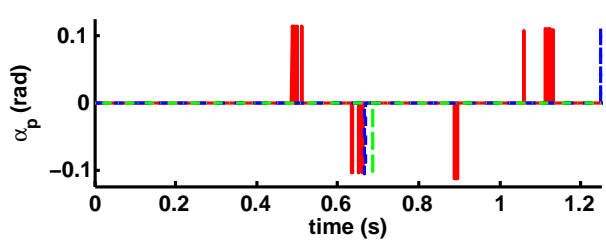

a. Planing

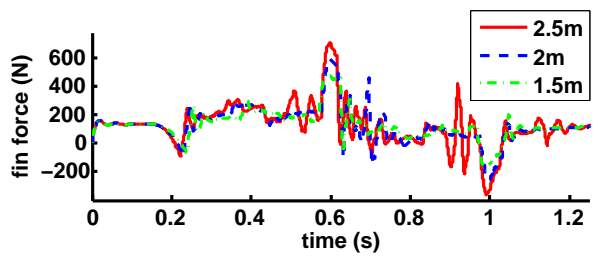

b. Actuator deflections

Figure 10. Tracking different amplitude maneuvers with predictive controller

ized variables improve stability with oscillation damping. The planing depth is not weighted high (1000). It is important to note that the output variable constraints are implemented as soft constraints, and planing depth constraint violations generate 10 times higher slack variable than other outputs. The slack variable weight is chosen to be $2.5 \times 10^{9}$. The actuator model $G_{a c t}=\frac{200}{s+200}$, and the disturbance model $G_{n}=\frac{0.1}{55.93}(R c-R) \frac{10^{6}}{s^{2}+2000+10^{6}}$ are the same as before.

The same $1.2 \mathrm{~s}$ reference trajectory on $z(t), w(t), \theta(t)$ and $q(t)$ is used. The results with the basic setup for 1.5,2, and $2.5 \mathrm{~m}$ amplitude maneuvers are shown in Figures 9 and 10.

The RHC reference tracking performance is less precise (Figure 12) than the pole placement controller, particularly on the signals with lower weights. The tradeoff is that planing occurs only for short periods with low depth (Figure 10). Tracking is achieved with low actuator 

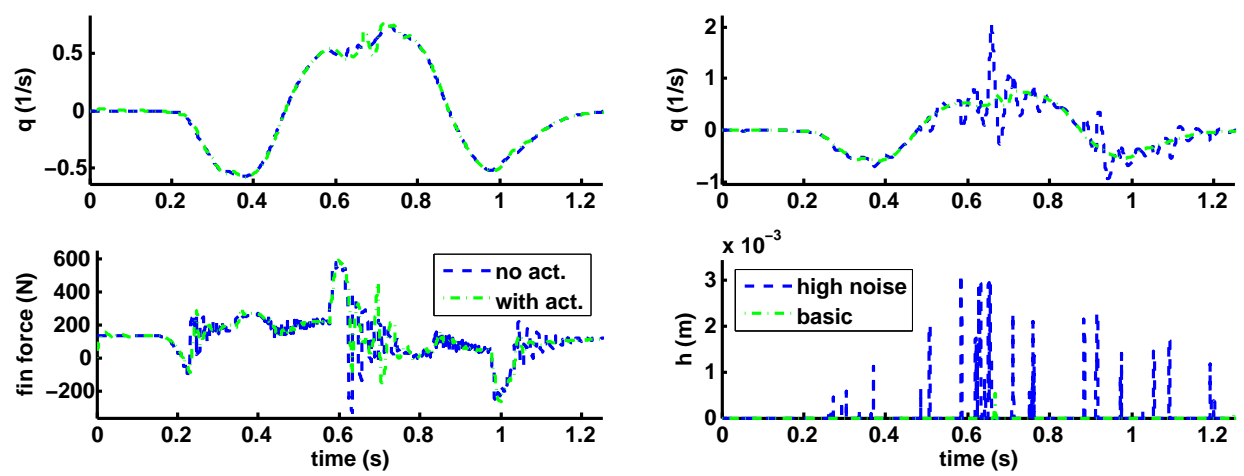

a. Actuator model

b. Disturbance magnitude

Figure 11. Sensitivity of RHC tracking performance

deflections, without oscillations. As the trajectory becomes more aggressive, planing occurs more frequently. This requires increased actuator usage, though the maximum planing depth, unlike in the pole placement case, is not increase with the trajectory amplitude. The overall control effort is significantly smaller than the pole placement design though at the expense of the state trajectories, especially the angle rate, being less smooth.

Uncertainty in delay time induces significant performance degradation because the bounds on constraining the maximum transom deviation from the cavity centerline are very tight. Uncertainty in the delay of $24 \mathrm{~ms}$ leads to oscillatory behavior, and larger control deflections are commanded due to the consequent uncertainty in the planing location. The closed-loop system becomes unstable around 10$15 \%(24-36 \mathrm{~ms})$ error in delay time.

The impact on tracking performance due to the addition of actuators, which are not addressed in the controller design, is shown in Figure 11. As one would expect, the performance is better if the actuator is perfect. Planing occurs for a very short time when actuators are included in the simulation. Assuming perfect actuators provide reduced oscillations, at the expense of high-rate control signals, what can significantly influence the cavity stability (Syrstad et al., 2005).

The disturbance magnitude has a strong influence on the performance. A comparison with a disturbance magnitude of 0.1 and 0.5 cavity gap is shown in Figure 11. As the disturbance magnitude increases, planing occurs more frequently and the immersion depth increases. This leads to larger control deflections and fast angle rate responses. The position tracking performance is not significantly affected by the disturbance level. 

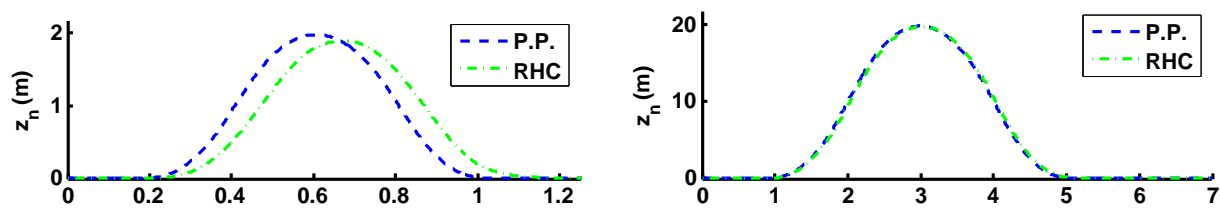

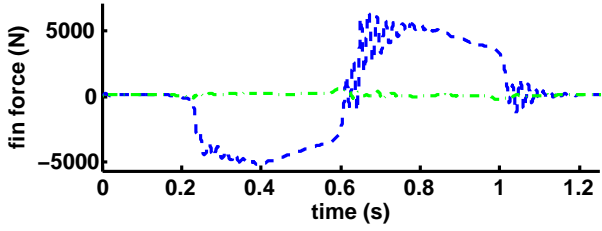

a. $2 \mathrm{~m}$ maneuver

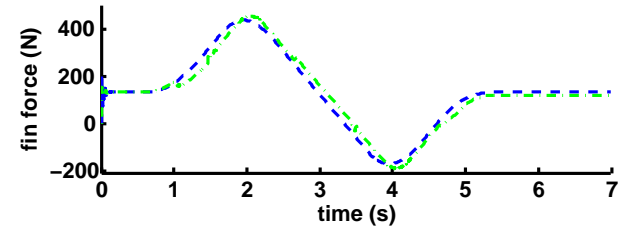

b. $20 \mathrm{~m}$ maneuver

Figure 12. Comparison of pole placement and predictive controller

The bandwidth of the cavity disturbance model also influences the closed-loop performance. Although limited information is available about the cavity wall smoothness, it is natural to assume that it is not perfect. The selected disturbance magnitude is $0.1\left(R_{c}-R\right)$ passed through a $1000 \mathrm{rad} / \mathrm{s}$ low-pass filter. The nominal simulation uses a second order filter $G_{n o m}(s)=\frac{0.1}{K_{n f}}(R c-R) \frac{10^{6}}{s^{2}+2000+10^{6}}$ which is normalized to provide approximately maximum $0.1(R c-R)$ magnitude signals. Two other disturbance filters are studied: a normalized first order $\left(K_{1} \frac{1000}{s+1000}\right)$ and a third order one $\left(K_{3} \frac{10^{9}}{s^{3}+3000 s^{2}+3 \cdot 10^{6} s+10^{9}}\right)$, they are comparable in point of all their poles are at $1000 \mathrm{rad} / \mathrm{s}$ and the maximum magnitude of the cavity disturbance is held constant. The closed-loop response with third-order disturbance filter planes longer, also causing larger angle rates. Hence, it indicates the importance of correct characterization of the cavity wall disturbance.

Longer maneuvers with higher amplitude excursions $(4 \mathrm{~s}, 20 \mathrm{~m})$ are also considered with the RHC design (Figure 12). As expected from the pole placement results, planing does not occur with the receding horizon approach. The state and control trajectories are very similar to the pole placement case. Figure 12 also shows the importance of planing avoidance, since the pole-placement controller commands unrealistically high actuator deflections in the short maneuver when planing occurs.

A simulation is performed with only a position reference signal, while all the other states are desired to be zero, to analyze how the constraints restrict the motion of the vehicle. Slight degradation in the position tracking performance is observed. The actuator deflection and all the vehicle state trajectories are very close to the original reference 

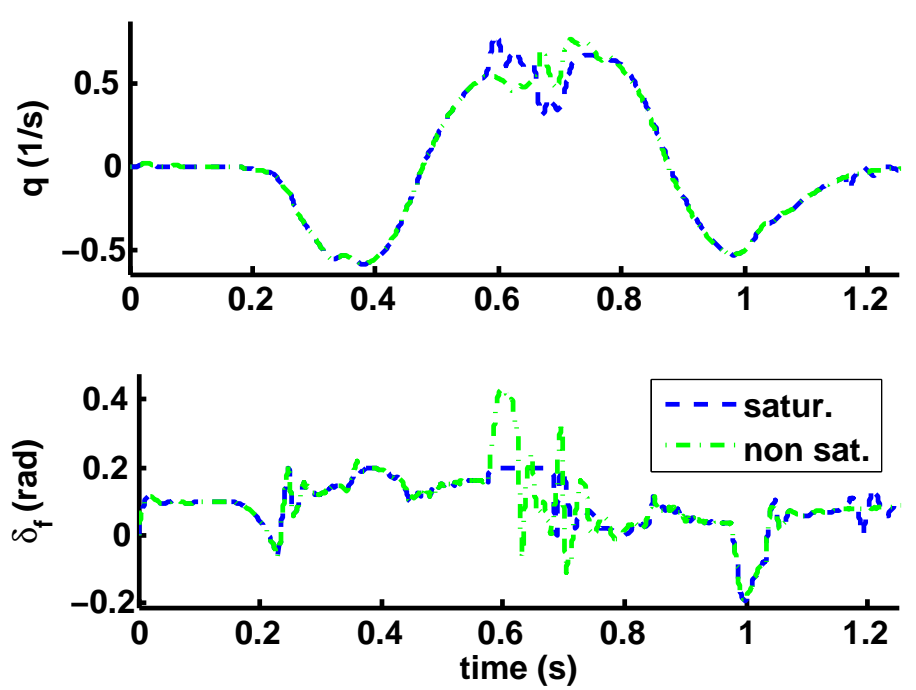

Figure 13. Tracking with hard actuator constraints with predictive controller $(2 \mathrm{~m}$ maneuver)

case. Hence, as was expected, planing avoidance represents a very tight constraint on the system.

The RHC scheme was implemented on the plant to aid in avoiding actuator saturations. As indicated in Figure 3, the control loop has two independent components. Therefore, direct constraint fulfilment is not possible. The controller performance is analyzed with hard actuator constraints on cavitator and fin deflections set to $0.2 \mathrm{rad}$ in Figure 13. Note that the fin deflection command increases to $0.4 \mathrm{rad}$ at $0.55 \mathrm{~s}$ but it is not allowed. The plant remains stable with slightly degraded performance, while the system with pole placement controller (Balas et al., 2006) becomes unstable with same conditions at this maneuver. However, if the trajectory becomes more aggressive, the tracking performance and/or the stability of the system become poor with hard actuator limitations using the RHC controller.

\section{Summary}

Two outer loop control strategies are implemented with a dynamic inversion controller for the HSSV. The main objective of the pole- placement design is to stabilize the vehicle and provide precise trajectory tracking commands, while the actuator deflections are not constrained. Stabilization and tracking are successfully demonstrated, and with selection of reference signals, planing was avoided in sufficiently large 
maneuvers. The pole-placement controller was insensitive to cavity disturbances, though the performance is strongly affected by the delay time. For certain cases, the pole-placement controller led to significant immersion into the fluid requiring high actuator deflections which resulted in increased drag on the hull and fins.

With the receding horizon approach, planing avoidance was successfully incorporated into the performance objectives at the expense of reduced tracking precision and higher sensitivity to cavity disturbances and delay information. The smaller immersion depth and actuator deflections led to significantly lower drag in all maneuvers. Although the approach relies heavily on the precision of the vehicle mathematical model, its beneficial properties make it a reasonable method for further development.

\section{Conclusion}

Supercavitation is a very promising way to increase the speed of underwater vehicles at the expense of a complicated vehicle architecture. Successful development of such system will require increased collaboration between fluid and control researchers. As an intermediate step, the control design challenges including delayed state dependency, nonlinearities, and switching with disturbed switching surface were analyzed. An inversion based control methodology with RHC extension was proposed for the 2-DOF mathematical model of the HSSV. An extensive comparison was made between a classical linear outer-loop controller and the receding horizon controller. The objective of planing avoidance was solved, for a limited operating range. Important aspects of the reference maneuvers were analyzed and sensitivity properties (a vulnerable point of dynamic inversion) were studied with respect to different cavity disturbances.

The next step of the analysis is to study the use of a single actuator for control (cavitator or fins), to understand the system tradeoffs. The ultimate goal remains the implementation of a three dimensional trajectory tracking controller on the HSSV test vehicle. It is likely that the controller for the high fidelity supercavitating model would require a gain-scheduled controller. This raises interesting issues with the design of dynamic inversion controller as the model parameters, like velocity or fin immersion, vary.

Furthermore, robust constraint fulfillment remains an open issue, which can be attempted to solve by further developments on the proposed receding horizon control based method. 


\section{Acknowledgments}

This work was funded by the Office of Naval Research, award number N000140110229, Dr. Kam Ng Program Manager.

\section{References}

Arndt, R., G. Balas, and M. Wosnik: 2005, 'Control of cavitating flows: A perspective'. Japan Society of Mechanical Engineers International Journal, 48(2): 334-341.

Ashley, S.: 2001, 'Warp drive underwater'. Scientific American, 42-51.

Balas, G., J. Bokor, B. Vanek, and R. Arndt: 2006, Control of Uncertain Systems: Modelling, Approximation and Design, Chapt. Control of High-Speed Underwater Vehicles, pp. 25-44, Lecture Notes in Control and Information Sciences. Springer-Verlag.

Balas, G., R. Chiang, A. Packard, and M. Safanov: 2005a, 'Robust Control Toolbox'. MUSYN Inc. and The MathWorks, Natick MA.

Balas, G. J., Z. Szabó, and J. Bokor: 2005b, 'Controllability of bimodal LTI systems'. submitted to: IEEE Transactions on Automatic Control.

Bemporad, A., M. Morari, and N. Ricker: 2005, Model Predictive Control Toolbox User's Guide. The Mathworks.

Çamlibel, M., W. Heemels, and J. Schumacher: 2004, 'On the controllability of bimodal piecewise linear systems'. In:Alur R, Pappas GJ (eds.) Hybrid Systems: Computationand Control LNCS 2993, Springer, Berlin, 250-264.

DARPA Advanced Technology Office: 2005, 'Underwater Express'. In: BAA06-13 Proposer Information Pamphlet (PIP).

Doyle, J., K. Glover, P. Khargonekar, and B. Francis: 1989, 'State-space solutions to standard $\mathcal{H}_{2}$ and $\mathcal{H}_{\infty}$ control problems'. IEEE Trans Auto Control 34:831-84\%.

Dzielski, J. and A. Kurdila: 2003, 'A Benchmark Control Problem for Supercavitating Vehicles and an Initial Investigation of Solutions'. Journal of Vibration and Control 9(7), 791-804.

Goel, A.: 2002, 'Control Strategies for Supercavitating Vehicles'. Master's thesis, University of Florida.

Kirschner, I., D. Kring, A. Stokes, and J. Uhlman: 2002a, 'Control strategies for supercavitating vehicles'. J Vibration and Control 8:219-242.

Kirschner, I., B. J. Rosenthal, and J. Uhlman: 2003, 'Simplified Dynamical Systems Analysis of Supercavitating High-Speed Bodies'. In: Fifth International Symposium on Cavitation (CAV2003). Osaka,Japan.

Kirschner, I. N., D. C. Kring, A. W. Stokes, N. E. Fine, and j. James S. Uhlman: 2002b, 'Control Strategies for Supercavitating Vehicles'. Journal of Vibration and Control 8, 219-242.

Kurdila, A. J., R. Lind, J. Dzielski, A. Jammulamadaka, and A. Goel: 2003, 'Dynamics and Control of Supercavitating Vehicles'. Technical report, Office of Naval Research Supercavitating High Speed Bodies Workshop.

Lin, G., B. Rosenthal, E. Abed, and B. Balachandran: 2004, 'Dynamics and Control of Supercavitating Bodies'. In: TECH2004.

Logvinovich, G.: 1972, 'Hydrodynamics of free-boundary flows'. translated from the Russian (NASA-TT-F-658), US Department of Commerce, Washington D.C.

Maciejowski, J.: 2002, Predictive Control with Constraints. Prentice Hall. 
Mayne, D., J. Rawlings, C. Rao, and P. Scokaert: 2000, 'Constrained model predictive control: stability and optimality'. Automatica (36), 789-814.

McFarlane, D. and K. Glover: 1992, 'A Loop Shaping Design Procedure using $\mathcal{H}_{\infty}$ Synthesis'. IEEE Trans Auto Control 37:759-769.

Safonov, M.: 1987, Modelling, Robustness and Sensitivity Reduction in Control Systems, Chapt. Imaginary-axis zeros in $\mathcal{H}_{\infty}$ optimal control, pp. 71-82. Springer-Verlag.

Shao, Y., M. Mesbahi, and G. Balas: 2003, 'Planing, switching and supercavitating flight control'. AIAA Guidance, Navigation and Control Conference, AIAA2003-5724.

Syrstad, J., M. Wosnik, G. Balas, and R. E. Arndt: 2005, 'Control of a SupercavityPiercing Fin'. In: 58th Annual Meeting of the Division of Fluid Dynamics.

Vanek, B., J. Bokor, and G. J. Balas: 2006a, 'High-Speed Underwater Vehicle Control'. AIAA Guidance, Navigation, and Control Conference, Keystone.

Vanek, B., J. Bokor, and G. J. Balas: 2006b, 'Theoretical aspects of High-Speed Underwater Vehicle Control'. American Control Conference, Minneapolis.

Vasin, A. and E. Paryshev: 2001, 'Immersion of a Cylinder in a Fluid Through a Cylindrical Free Surface'. Fluid Dynamics 36(2), 169-177. translated from Russian. 